\title{
The Enrichment of Galaxies by Quasar Outflows
}

\author{
G. Chartas ${ }^{1}$, W. N. Brandt ${ }^{1}$, S. C. Gallagher ${ }^{2}$, G. P. Garmire ${ }^{1}$ \\ 1 Pennsylvania State University, USA \\ 2 University of California, Los Angeles, USA
}

\begin{abstract}
Our recent Chandra and XMM-Newton observations of the gravitationally lensed broad absorption line quasars (BALQSOs) APM $08279+5255$ and PG $1115+080$ have provided new insights into the structure of quasar outflows and the enrichment of their host galaxies by quasar winds (Chartas et al. 2002, 2003). Our spectral analysis of these observations suggests the presence of X-ray BAL material accelerated to relativistic velocities of up to $\sim 0.4 c$, considerably larger than the observed velocities of the UV BAL absorbers in these objects. We present constraints on the quasar mass-outflow rates based on the observed relativistic outflow velocities and locations of the X-ray BAL material.
\end{abstract}

\section{Introduction}

It is commonly accepted that most quasars contain energetic outflows of ionized gas emerging from their accretion disks at speeds ranging from $\sim 5,000$ $30,000 \mathrm{~km} \mathrm{~s}^{-1}$ (e.g., Turnshek et al. 1988; Weymann et al. 1991). An estimate of the mass-outflow rate may be used to evaluate the contribution of outflowing winds in distributing accretion-disk material into the vicinity of the quasar central engine and the host galaxy over the life time of a quasar. Constraining this rate is also important for understanding the connection between black hole and bulge growth in the host galaxy (e.g., Fabian 1999). Present X-ray observations of BALQSOs APM $08279+5255$ and PG $1115+080$ have allowed us to constrain the properties of the outflowing ionized, outflowing X-ray absorbers and infer the contribution of these winds to the total mass-outflow rates.

\section{Observations and Results}

APM $08279+5255$ is a $z=3.91$, IR-bright $(I=14.6)$ BALQSO that was observed with ACIS (Garmire et al. 2003) onboard the Chandra X-ray Observatory on 2000 October 11 and 2002 February 24 for $9.2 \mathrm{ks}$ and $88.8 \mathrm{ks}$, respectively. A 100 ks observation of APM $08279+5255$ was also performed with XMM-Newton 1.8 weeks (proper-time) after the Chandra observation. PG $1115+080(z=1.72$, $B=16.1$ ) is a quadruply lensed mini-BALQSO that was observed for $62.6 \mathrm{ks}$ on 2001 Nov 25 with XMM-Newton. It was also observed with Chandra on 2000 June 2 and 2000 Nov 3 for $26.8 \mathrm{ks}$ and $10.0 \mathrm{ks}$, respectively. 
Spectral analyses indicate the presence of relativistic outflows in these BAL QSOs imprinted as resonant X-ray absorption lines of highly ionized iron (Fe XXV $\mathrm{K} \alpha$ and/or Fe XXVI K $\alpha$ ). The energies of the observed absorption features suggest the presence of X-ray BAL material outflowing from the central source at velocities of $\sim 0.2 c$ and $\sim 0.4 c$ for APM $08279+5255$ and $\sim 0.1 c$ and $\sim 0.34 c$ for PG $1115+080$. Significant variability of the energies and widths of the BALs in APM $08279+5255$ are presented in Figure 1; this confirms the intrinsic origin of the BAL features. The detection of variability between the two observations of APM $08279+5255$ over a timescale of 1.8 weeks (proper-time) suggests that these absorbers are most likely launched at relatively small radii of $\lesssim$ $10^{16}\left(M_{b h} / 10^{8} M_{\odot}\right)^{1 / 2} \mathrm{~cm}$.

The estimated mass-outflow rate for $r / d r$ ranging from $1-10$ is $1-20 \mathrm{M}_{\odot}$ $\mathrm{yr}^{-1}$ (see Fig. 1), where $r$ is the distance from the black hole to the absorber and $d r$ is the thickness of the absorber. The mass-outflow velocities in APM 08279+5255 and PG $1115+080$ significantly exceed the escape velocities of galaxies and clusters of galaxies, and therefore quasar winds may play a significant role in the enrichment of the IGM. In particular, the estimated mass-outflow rates in these two BALQSOs suggest that over a quasar's lifetime as much as a few $10^{9} \mathrm{M}_{\odot}$ of high-metallicity material could be recycled via energetic winds into the ISM and IGM.
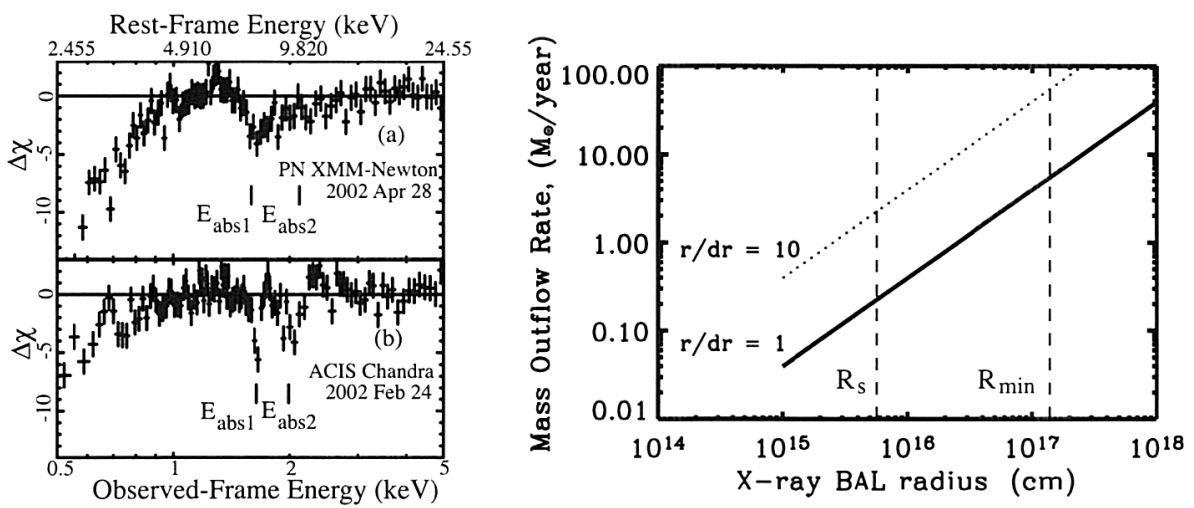

Figure 1. $\quad \Delta \chi$ residuals between the best-fit Galactic absorption and power-law model and (a) the XMM-Newton PN spectrum and (b) the Chandra ACIS spectrum of APM 08279+5255. (right) : Estimate of mass-outflow rate versus radius for APM $08279+5255 . R_{s}$ and $R_{\text {min }}$ are the Schwarzschild and minimum launching radii, respectively.

Acknowledgments. We acknowledge support from NASA grants NAS801128 (GPG), NAG5-9949 (GC), NAG5-13035 (WNB) and NAG5-9932 (WNB). 


\section{References}

Chartas, G., et al. 2002, ApJ, 579, 169

Chartas, G., Brandt, W. N., \& Gallagher, S. C., 2003, ApJ, 595, 85

Fabian, A. C. 1999, MNRAS, 308, L39

Garmire G. P., et al. 2003, SPIE, 4851, p28

Turnshek, D. A., et al. 1988, ApJ, 325, 651

Weymann, R. J., et al. 1991, ApJ, 373, 23 